\title{
An atomistically informed kinetic Monte Carlo model of grain boundary motion coupled to shear deformation
}

\author{
M. Prieto-Depedro, I. Martin-Bragado, J. Segurado
}

\section{A B S T R A C T}

The shear coupled motion of grain boundaries (GBs) is modelled by using two different atomistic simulation techniques: molecular dynamics (MD) and kinetic Monte Carlo (KMC). MD simulations are conducted to identify the elementary mechanisms that take place during the coupled motion of GBs. This process is described on the one hand, in terms of the geometrical approach of the dislocation content in the boundary; and on the other hand, by the thermodynamics of the dislocation passage, shown as a thermal activated pro-cess. Relevant MD output is extended into a KMC model that considers the GB migration as a result of a sequence of discrete rare events. The independent motion of each structural unit forming the boundary conforms a single event, having a rate per unit of time to move to the next stable position computed according to the transition state theory. The limited time scale of classical MD is overcome by KMC, that allows to impose realistic deformation velocities up to $10 \mu \mathrm{m} / \mathrm{s}$.

Keywords:

A. Grain boundaries

A. Dislocations

A. Stress relaxation

C. Numerical algorithms

B. Metallic material

\section{Introduction}

Grain boundaries play an important role in determining the mechanical properties of nanocrystalline (nc-) materials (Hirth, 1972), e.g. enhancement of the initial resistance to yielding in nc-aggregates by the presence of GBs (Wulfinghoff et al., 2013). Several investigations have been carried out in order to understand the GB structure (Farkas, 2000), thermodynamics (Mishin et al., 2010) and related deformation mechanisms (Bieler et al., 2009; Dahlberg et al., 2013).

In particular, atomistic simulations have provided a further insight into the microscopic mechanisms and fracture nucleation at interfaces under applied mechanical loads. Shear behaviours of GBs have been studied in detail, and grain boundary (GB) motion coupled to shear deformation has been evidenced to be an important mode of plastic deformation, as a dominant behaviour or competing with other GB mechanisms in a wide range of temperatures, such as GB sliding (Du et al., 2010; Schäfer and Albe, 2012; Warner et al., 2006) or dislocation mediated slips (Sansoz and Molinari, 2005; Tschopp and McDowell, 2008; Spearot et al., 2007; Ohashi et al., 2009).

Basically this mechanism is described through the coupling of the GB to mechanical loads which in turn induces a shear deformation in the crystal region swept by the motion. Stress-induced GB migration was first observed in symmetrical low-angle GBs (LAGBs) in Zn bicrystals (Li et al., 1953). Read and Shockley (1950) determined the mechanism of this motion to be due to the collective glide of the array of parallel edge dislocations forming the LAGB. This mechanism was limited to boundaries with low tilt angle where the core of individual dislocations could be resolved. However, this model has been 
extended to high- angle GBs (HAGB) in the case of metals. Goryaka et al. (2009) confirmed experimentally the coupled motion of GBs for both low- and high-angle symmetrical tilt GBs with a misorientation angle within 0-90 in Al bicrystals. Also, computer simulations evidence that the coupling between normal boundary motion and the rigid translation of the adjacent grains can occur for HAGBs (Cahn et al., 2006; Frolov, 2014; Huang et al., 2014). The coupling effect was proposed to be a generic property of many atomically ordered GBs in the unified approach reported by Cahn and Taylor (2004) and confirmed over a large catalogue of 388 GBs (Homer et al., 2013).

To date, GB shear-coupled motion has been subjected to several studies to explore the impact of this mechanism in the mechanical properties of nc- materials, as well as its relation to other deformation processes (Tucker et al., 2010; Rajabzadeh et al., 2014; Goryaka et al., 2011; Berbenni et al., 2013a). The major challenge relies on the multiscale modelling, as discussed by Berbenni et al. (2013b) where a complete micromechanical model based on molecular dynamics (MD) simulations is proposed. Taupin et al. (2014) describe the shear coupled boundary migration in a continuous manner by using an elasto-plastic theory of disclination and dislocation fields, and results are found to be in good agreement with atomistic simulations and experiments.

In this paper, we continue to investigate the dynamics of this phenomenon within a multiscale framework for its comprehensive understanding. Two different atomistic simulation techniques are used throughout this work. On the one hand, MD simulations are used to identify the elementary mechanisms of GB migration, and on the other hand, we have extended the outcome of MD simulations to a kinetic Monte Carlo (KMC) model. KMC models the shear coupled motion of GBs based on the thermodynamics of the dislocation motion, in terms of individual resolved cores forming the GB. The main goal is to be able to reproduce the MD simulation results at a reduced computational cost by means of KMC. We apply this multiscale approach in bicrystals containing one individual [001] symmetrical tilt GB in $\mathrm{Ni}$ in a range of misorientation angles between $0^{\circ}$ and $90^{\circ}$.

The structure of the present paper is the following. In Section 2 a complete description of the dynamics of coupling and the geometric characterization of GBs are introduced. MD simulations are carried out in different temperature regimes and reported in Section 3. To reach our final goal of extending the MD output, the main concern before moving to KMC is to validate such output. Due to the lack of experimental data, in Sections 3.2 and 3.3 simulated values are compared in detail to theoretical models previously illustrated. The minimum energy path of the shear-coupled GB motion is computed using the nudged elastic band method in Section 3.4, which is the most relevant input in the KMC method. Within the multiscale approach, the latter is proposed in Section 4. Numerical examples of KMC are presented in Section 4.2, where results are contrasted to previously shown MD results.

\section{Dynamics of shear-coupled motion of grain boundaries and coupling modes}

The simple model proposed by Ivanov and Mishin (2008) assumes the coupled GB motion as a motion through a periodic landscape. Following this approach, the system has a set of equivalent stable states which are separated by an energy barrier. At $0 \mathrm{~K}$, in the absence of any external force applied, the GB is trapped in a particular equilibrium position. By applying a shear stress through imposing a parallel velocity $\left(v_{\|}\right)$, the GB is elastically deformed and when a critical value $\tau_{c}^{0}$ is reached, the relevant barrier vanishes to zero and the GB makes a transition by increments of $H$. This normal motion of the GB occurs simultaneously with the rigid-body translation of the adjacent grains $(S)$, producing a permanent shear deformation of the lattice and the subsequent drop of the stress. The ratio $\beta=S / H$ is called coupling factor, discussed later. The first stress relaxation is followed by a new elastic loading step until the critical stress is reached again, and the GB makes another step, see Fig. 1, identified as a stick-slip behaviour.

At finite temperature, the GB could move before the critical stress is reached. Thermal fluctuations assist the GB to overcome the energy barrier, so critical stress $\tau_{c}$ is then expected to decrease with temperature. This decreasing is intensified as temperature increases, and at a fixed $v_{\|}$the peak stress is expected to be linear in $T^{2 / 3}$ as (Ivanov and Mishin, 2008):
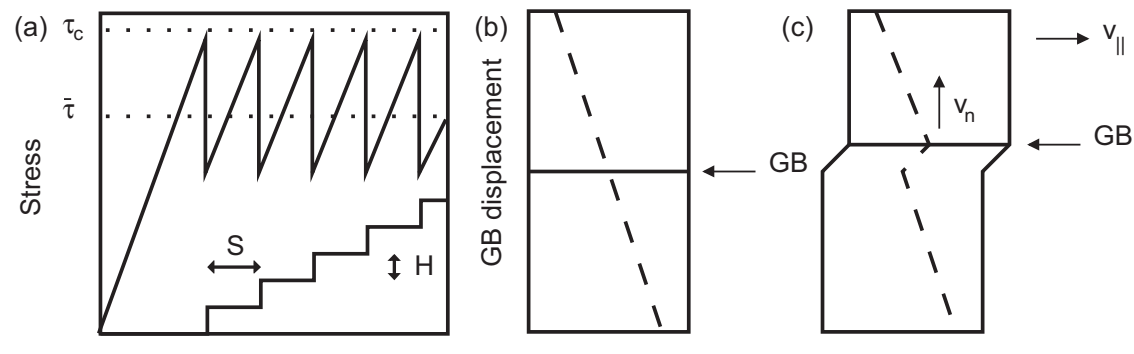

Grain translation

Fig. 1. Stick-slip behaviour. (a) In a perfect coupled motion, the normal motion of GB by increments of $\mathrm{H}$ is accompanied by rigid translation of the grains by increments of S. After each jump of the GB to the next stable position the stress drops. (b) Initial configuration of the system and (c) sheared lattice. 


$$
\tau_{c}=\tau_{c}^{0}-B T^{2 / 3}
$$

being $B$ a constant dependent on the attempt frequency and other factors. As temperature continues to increase, spontaneous movements of the GB may occur, and the stick-slip behaviour can be destroyed or replaced by other dominant mechanisms, such as sliding events.

At a fixed temperature, critical stress is expected to increase with the imposed grain translation velocity since the time to overcome the barrier is reduced. This variation is given by (Sang et al., 2001; Garg, 1995):

$$
v_{\|}=\frac{\tau_{c}^{0}}{\eta}\left(1-\frac{\tau_{c}}{\tau_{c}^{0}}\right)^{1 / 2} \exp \left[-\frac{\left(1-\tau_{c} / \tau_{c}^{0}\right)^{3 / 2} E_{0}}{k_{B} T}\right],
$$

where $\eta$ represents an effective friction coefficient, $k_{B}$ is the Boltzman constant and $E_{0}$ is the energy barrier in the absence of temperature. This variation was tested by using accelerated MD methods that allow to cover a wide range of velocities that are not accessible by classical MD (Mishin et al., 2007).

Regarding the GB crystallography, GBs consists of identical structural units. When resolving individual cores is possible, GBs are identified as an array of parallel edge dislocations. As a result, GB shear coupled motion is understood as a result of the passage of the dislocation content inducing itself plastic deformation of the lattice (Read and Shockley, 1950).

To define the dislocation content of a GB the Frank-Bilby equation (FBE) is solved (Frank, 1953; Bilby, 1955), and the coupling factor is determined to be related to GB interfacial Burgers vector and slip plane. The crystal symmetry leads to the multiplicity of the dislocation content, and so to different coupling factors. By exploring the two possible solutions for the [001] tilt GBs considered in the present work, two possible Burgers vectors are identified, and as a result two different coupling modes are predicted, later acting as KMC inputs. When the misorientation angle $(\theta)$ tends to 0 , the set of dislocations corresponds to Burgers vector $\mathbf{b}=$ [100], being the Frank-Bilby dislocation slip planes (100). This model is extended to GB with $\theta$ approaching $90^{\circ}$, with Burgers vector $\mathbf{b}=-1 / 2[110]$ and glide along (110) planes. Assuming the dislocations move in increments of $\mathbf{b}$, the two branches of the misorientation dependence of $\beta$ are obtained (Cahn et al., 2006). There is one branch called $\langle 100\rangle$, for $\theta \rightarrow 0$, given by:

$$
\beta_{\langle 100\rangle}=2 \tan \left(\frac{\theta}{2}\right) .
$$

The other branch corresponds to $\theta \rightarrow 90$, as:

$$
\beta_{\langle 110\rangle}=-2 \tan \left(\frac{\pi}{4}-\frac{\theta}{2}\right) .
$$

In between, boundaries can move according to either of the two possible modes. These modes compete with each other, existing a transition between them at a critical $\theta$ depending on the temperature. The expansion of the $\langle 110\rangle$ mode as temperature decreases was observed in Cu bicrystals (Cahn et al., 2006), even it may be the only active mode at $0 \mathrm{~K}$ for the whole range of $\theta$. To activate the $\langle 100\rangle$ mode, the mirror symmetry due to equivalent row translations by lattice vectors $1 / 2[001]$ and $1 / 2[00 \overline{1}]$ has to be broken, and this is only feasible at finite temperatures when the GB develops ledges and steps assisting in breaking such symmetry (Berbenni et al., 2013b). Boundaries tend to move in the most energetically favored mode, and as a consequence those boundaries corresponding to $\langle 100\rangle$ mode move instead according to $\langle 110\rangle$ at low temperatures. At this point, it is obvious that the switch between modes is related with a change in the dislocation content. Note that the two coupling modes have different signs, and then GBs are expected to move in opposite directions depending on the active mode.

\section{Molecular dynamics}

\subsection{Computational procedure}

Atomistic simulations are performed using an embedded-atom method potential fit to a large database including both experimental and first-principles data for Ni (Mishin et al., 1999). A series of [001] symmetrical tilt GBs with different misorientation angle are created, see Table 1 . Two separated crystals with the desired crystallographic orientation are joined along a plane normal to $x_{2}$-direction, see Fig. 2 . The simulation block contains two grains forming a bicrystal configuration with only one planar GB in the middle. Periodic boundary conditions are imposed in the parallel directions to the GB plane, $x_{1}$ and $x_{3}$. In the normal direction, $x_{2}$, dynamic atoms are sandwiched between two slabs whose thickness is about twice the cutoff radius of the interatomic potential. Atoms at the slabs are held fixed in their perfect lattice positions and do not participate in computing data from the simulations. Each symmetrical tilt GB is identified by the indices (hkl) of the GB plane and by the reciprocal value of the total number of coincidence sites, $\Sigma$ (Kronenberg and Wilson, 1947). Throughout the text, the notation will be $\Sigma(\mathrm{hkl})$.

MD simulations are carried out using LAMMPS (Plimpton, 1995) simulator. As a first step, the ground-state structure of each GB is determined by static minimization of the potential energy of the whole system. The canonical NVT ensemble (number of particles, volume and constant temperature) is used to perform the simulations. The time step is $0.2 \mathrm{fs}$. The shear 
Table 1

Characteristics of [001] symmetrical tilt GBs in Ni. The ideal coupling factor predicted by geometric approaches and from MD simulations is unitless. The effective elastic shear modulus obtained by the transformation field analysis (TFA) and MD results is in GPa.

\begin{tabular}{|c|c|c|c|c|c|c|}
\hline Boundary & $\theta$ & Mode & $\beta_{\text {ideal }}$ & $\beta_{M D}$ & $\mu_{T F A}^{e f f}$ & $\mu_{M D}^{e f f}$ \\
\hline$\Sigma 25(710)$ & 16.26 & $\langle 100\rangle$ & 0.286 & 0.305 & 117.51 & 119.40 \\
\hline$\Sigma 37(610)$ & 18.92 & $\langle 100\rangle$ & 0.333 & 0.372 & 115.03 & 122.02 \\
\hline$\Sigma 13(510)$ & 22.62 & $\langle 100\rangle$ & 0.400 & 0.423 & 111.15 & 113.30 \\
\hline$\Sigma 17(410)$ & 28.07 & $\langle 100\rangle$ & 0.500 & 0.507 & 104.71 & 107.76 \\
\hline$\Sigma 53(720)$ & 31.89 & $\langle 110\rangle$ & -1.111 & -1.160 & 99.83 & 95.85 \\
\hline$\Sigma 5(310)$ & 36.87 & $\langle 110\rangle$ & -1.000 & -1.004 & 91.42 & 93.27 \\
\hline$\Sigma 5(210)$ & 53.13 & $\langle 110\rangle$ & -0.667 & -0.673 & 72.60 & 66.45 \\
\hline$\Sigma 17(530)$ & 61.93 & $\langle 110\rangle$ & -0.500 & -0.545 & 63.43 & 57.88 \\
\hline$\Sigma 13(320)$ & 67.38 & $\langle 110\rangle$ & -0.400 & -0.430 & 58.81 & 53.10 \\
\hline$\Sigma 25(430)$ & 73.74 & $\langle 110\rangle$ & -0.286 & -0.259 & 54.59 & 47.12 \\
\hline$\Sigma 61(650)$ & 79.61 & $\langle 110\rangle$ & -0.182 & -0.186 & 51.88 & 46.68 \\
\hline
\end{tabular}

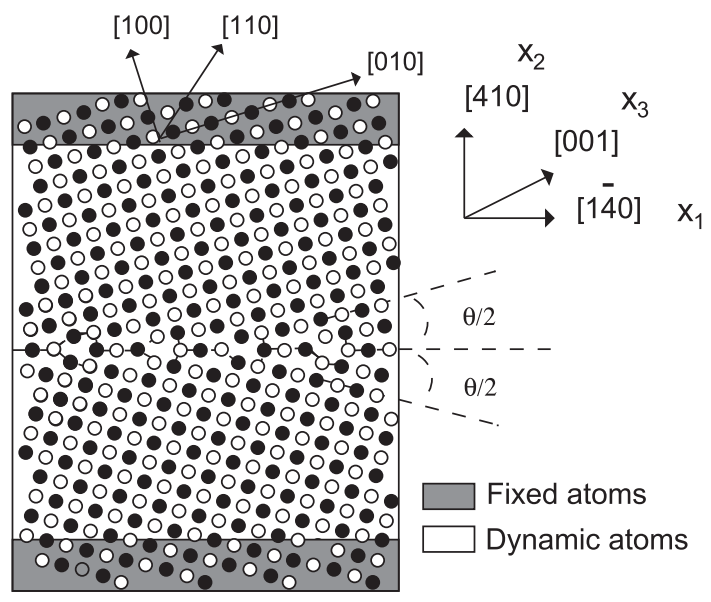

Fig. 2. Description of the simulation block for the $\Sigma 17(410) \mathrm{GB}$ with a misorientation angle of $\theta=28.07^{\circ}$. Open and filled circles represent atoms in alternated planes along $x_{3}$-direction. Structural units forming the GB are outlined. Grey regions contain fixed atoms.

rate applied in the top slab is given by $v_{\|}=\dot{E} L$, where $\dot{E}$ is the shear strain rate $\left(10^{8} \mathrm{~s}^{-1}\right)$ and $L$ is the length of the box containing unconstrained atoms. The imposed grain translation velocity results about $1 \mathrm{~m} / \mathrm{s}$, well above the shear velocities under real conditions due to the time scale limitations of classical MD. The stress tensor is averaged over all dynamic atoms using the standard virial expression and is constantly monitored during the simulation. GB position is tracked using common neighbour analysis (CNA) (Faken and Jonsson, 1994).

\subsection{Shear coupled motion at low temperatures}

Hereinafter, this work is mainly focused on the $\Sigma 17(410)$ symmetrical tilt GB (STGB). Its behaviour at different temperature regimes covers all the mechanisms to be described. Coupled motion is studied near $0 \mathrm{~K}$ : the upper slab is rigidly displaced with small increments, and after each one the system is fully relaxed while maintaining the constraint. Fig. 3 reports the shear stress and GB displacement variation as a function of the strain for $\Sigma 17(410)$. The GB does not move until the critical stress $\tau_{c}^{0}$ is reached, being each peak of stress exactly correlated with an increment of the GB motion. The $\tau_{c}^{0}$ MD output is transferred as KMC data in Section 4.

Every loading elastic step is associated with the same shear modulus $(\mu)$, obtained from the slope of the stress-strain curves. As discussed later, $\mu$ is also a relevant input in KMC simulations, and it is noteworthy to check if the interatomic potential is reliable for the computation of the elastic constants of the bicrystal structures. As a consequence, this magnitude has been also calculated theoretically using the transformation field analysis (TFA), first proposed by Dvorak (1990) and well described by Franciosi and Berbenni (2008), see Table 1.

According to this approach (see Appendix A), the effective shear modulus is determined to be:

$$
\left.\mu^{e f f}=\frac{1}{2} \quad C_{66}^{I}-\frac{\left(C_{62}^{I}\right)^{2}}{C_{22}^{I}}\right)+\frac{1}{2}\left(C_{66}^{I I}-\frac{\left(C_{62}^{I I}\right)^{2}}{C_{22}^{I I}}\right),
$$




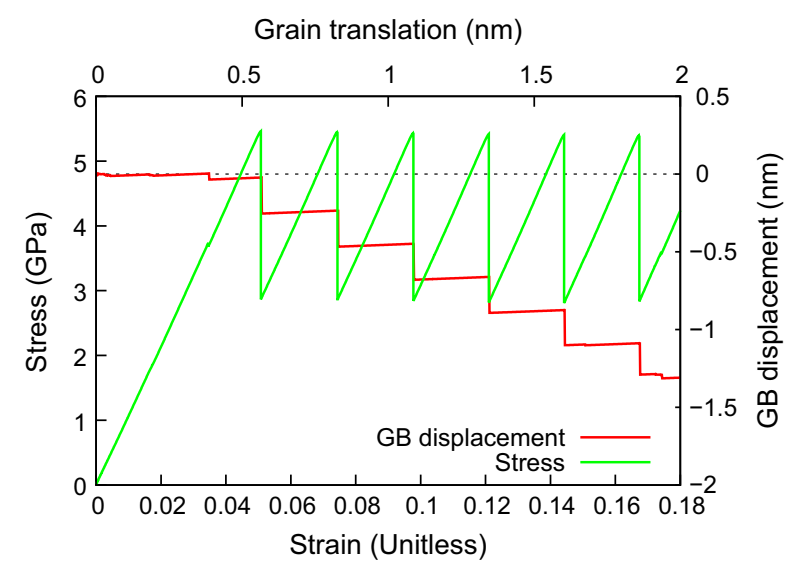

Fig. 3. Stress-Strain and GB displacement during coupled motion of the $\Sigma 17(410)$ at $0 \mathrm{~K}$ with $v_{\|}=1 \mathrm{~m} / \mathrm{s}$. The perfect coupled motion is clearly observed in the absence of temperature. The critical stress and the effective shear modulus are identified, being 5.45 GPa and 107.76 GPa, respectively.

being $C_{66}^{I}, C_{62}^{I}$ and $C_{22}^{I}$, and $C_{66}^{I I}, C_{62}^{I I}$ and $C_{22}^{I I}$ the anisotropic elastic constants of constituent crystals I and II, respectively, obtained according to Eqs. (A.1) and (A.2).

Turning to the coupling modes, Fig. 4 displays the dependence of $\beta$ with the misorientation angle. Values of $\beta_{M D}$ are calculated as the ratio between $S / H$. At $0 \mathrm{~K}$ all the GBs move according to the $\langle 110\rangle$ mode as expected, which could be explained by considering the discrete dislocation model of coupling at low temperatures, where the critical stress for the GB motion is related to the Peierls-Nabarro stress for the glide of the array of parallel edge dislocations (Hull and Bacon, 1965). PeierlsNabarro stress for the two set of dislocations could be compared by checking the $\gamma$ surfaces corresponding to each slip plane in the absence of temperature. A $\gamma$ surface represents the extra crystal energy per unit area of a generalized stacking fault, obtained by rigidly displace the two halves of a crystal by a vector parallel to such relevant slip plane (Hull and Bacon, 1965). Studies on Cu bicrystals reported by Cahn et al. (2006) determined that higher energy barriers lead to more compact dislocation core and larger Peierls-Nabarro stress. Energy for $\{110\}\langle 110\rangle$ and $\{100\}\langle 100\rangle$ slip systems have been calculated in Ni by rigidly displacing the two halves of a crystal on such slip planes, resulting in values of 1.15 and $2.12 \mathrm{~J} / \mathrm{m}^{2}$, respectively. These $\gamma$ energies should be used as a prediction since complex atomic movements may not be included in the Peierls-Nabarro model, as is the case of the dissociation of the [100] dislocations at this temperature regime, involving screw and edge components.

\subsection{Shear coupled motion at medium temperatures}

At low and medium temperatures, the stop-and-go character is still the dominant response of the GB under simple shear. Fig. 5 illustrates the stress behaviour during coupling motion at $500 \mathrm{~K}$ for $\Sigma 17(410)$, showing the decreasing of the critical stress due to thermal fluctuations. Before doing the final movement, intermediate jumps are also observed, those are understood as a weak stability of the GB at this temperature (Mishin et al., 2007). The frequency of these reverse jumps increases with temperature, and GBs move in a more stochastic manner.

As temperature continues to increase, the shear stress does not display a clear stick-slip behaviour anymore, as illustrated in Fig. 6 for $\Sigma 17(410)$ at different temperatures. The stress is replaced by random noise with some average value,

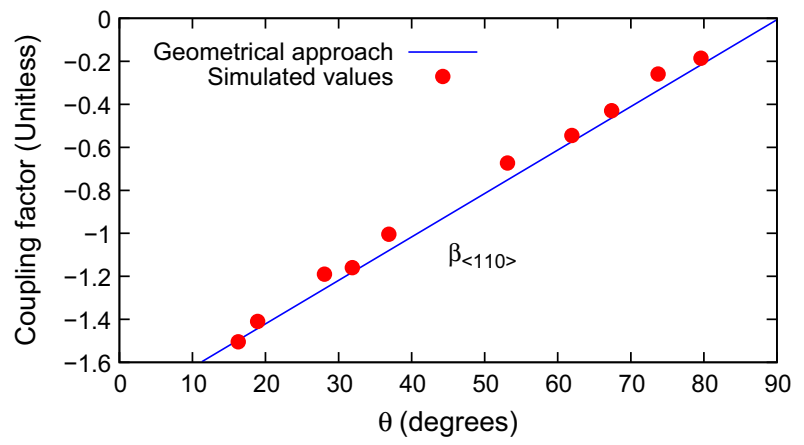

Fig. 4. Coupling factor obtained near $0 \mathrm{~K}$ by MD simulations is identified with red dots, and the branch of the $\langle 110\rangle$ mode given by Eq. (4) is represented by a blue line. (For interpretation of the references to colour in this figure legend, the reader is referred to the web version of this article.) 


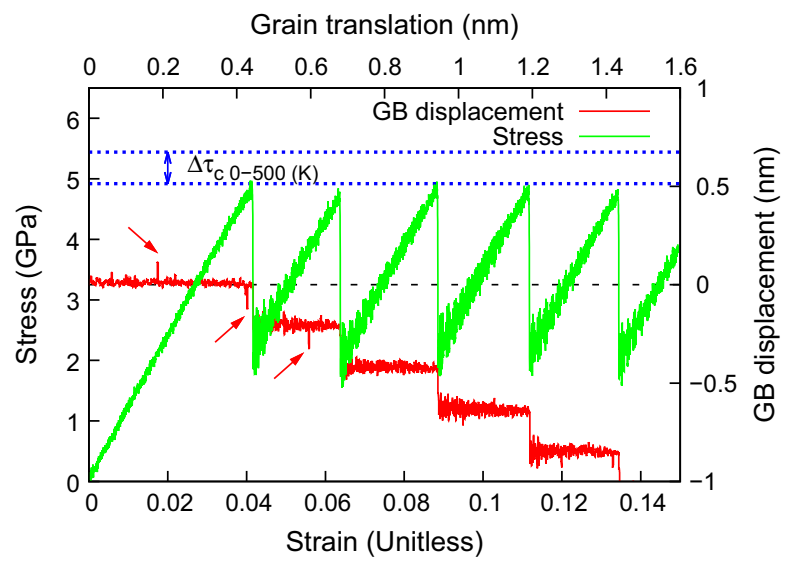

Fig. 5. Stress-strain and GB displacement during coupling motion of $\Sigma 17(410)$ at $500 \mathrm{~K}$ with $v_{\|}=1 \mathrm{~m} / \mathrm{s}$. Dashed lines represent the decrease of the critical stress with temperature respect to $\tau_{c}^{0}$. Arrows indicate reverse jumps of the GB.

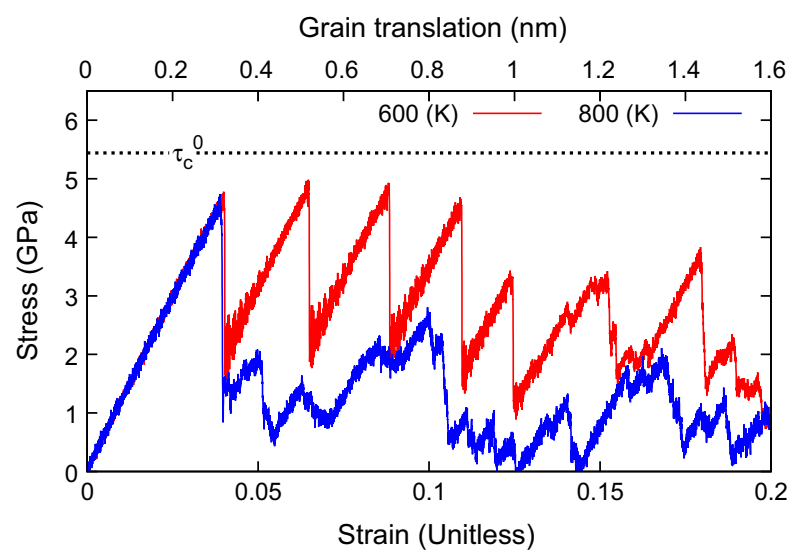

Fig. 6. Stress-strain curves during the coupled motion of $\Sigma 17(410)$ at $600 \mathrm{~K}$ and $800 \mathrm{~K}$, identified with red and blue lines respectively. The critical stress at $0 \mathrm{~K}$ is identified by a dashed line. (For interpretation of the references to colour in this figure legend, the reader is referred to the web version of this article.)

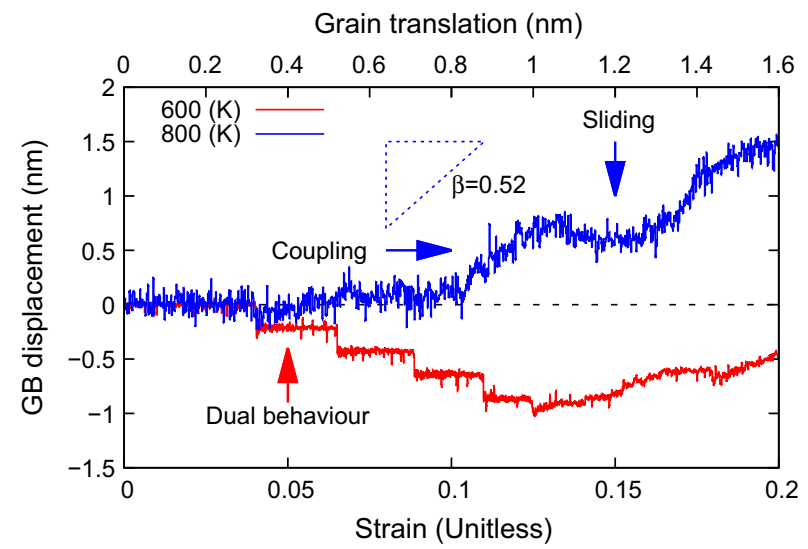

Fig. 7. GB displacement during the coupled motion of $\Sigma 17(410)$ at $600 \mathrm{~K}$ and $800 \mathrm{~K}$, identified with red and blue lines respectively. (For interpretation of the references to colour in this figure legend, the reader is referred to the web version of this article.)

$\bar{\tau}_{c}$. This behaviour indicates a transition to a different response of the GB. It is interesting to examine the mechanisms existing at these temperatures. Despite describing such mechanisms is out of the scope of the present paper, modelling the coupling effect by the KMC method requires the knowledge of the temperature regimes at which the pure coupling is not observed anymore. 
Fig. 7 illustrates the GB displacement as function of the strain for 600 and $800 \mathrm{~K}$. Red line shows an overlap area in which both modes are observed. As shown in Fig. 8, after n-migration events according to the $\langle 110\rangle$ mode, there is a switch to the $\langle 100\rangle$ mode related to a change in the dislocation content, observed as an abrupt transition. This behaviour is only observed for $\theta<30^{\circ}$, within a temperature range between 600 and $900 \mathrm{~K}$, specific to each GB. All the GBs identified as $\langle 100\rangle$ in Table 1 moves according to this mode once the activation stress is reached. Being known such GBs, as well as the temperature at which the second mode is activated (higher than $800 \mathrm{~K}$ for all of them), the Burgers vector to be introduced into KMC is defined as shown in the following sections.

Returning to Fig. 7, at $800 \mathrm{~K}$ GB coupled motion begins to be occasionally interrupted by sliding events, identified by the blue line. Grain translation is not accompanied by normal motion of the GB. Between sliding events, the GB continues to move in a coupled manner, with $\beta=0.52$, in good agreement with predicted value shown in Fig. 8, $\beta=0.50$ for $\Sigma 17(410)$ $\theta=28.07^{\circ}$. In this case, the coupling factor is obtained from the slope of the correlation between grain translation and normal motion of the GB, since at this temperature, it is not possible to measure the ratio between $S$ and $H$. The frequency of such sliding events increases with temperature, and the average normal velocity decreases. At a given temperature, approaching the melting point, $T_{m}=1728 \mathrm{~K}$ (Turley and Sines, 1971), GB shear-coupled motion is expected to be completely destroyed.

To understand the nature of the transition between these different mechanisms, $\Sigma 17(410)$ simulation results have been fit to Eq. (1), proposed by Ivanov and Mishin (2008) to describe the temperature dependence of the peak stress within the stick-slip regime. Fig. 10 shows the linear dependence on $T^{2 / 3}$ of the stress averaged over the entire simulation time, 15 stick-slip processes. The linearity indicates that the stick-slip behaviour is the dominant mechanism in this temperature regime. It should be noticed that Eq. (1) has been obtained assuming $\tau_{c}$ tends to $\tau_{c}^{0}$. However, at temperatures $600 \mathrm{~K}$ and higher, $\tau \nrightarrow \tau_{c}^{0}$, and the average stress deviates from the linear fit. Such deviation indicates the transition to other mechanical response of the GB.

\subsection{Nudged elastic band calculations}

As described in Section 2, GB migration is a thermal activated process. GBs are described in terms of the energetic characteristics of the elementary steps that take place during the coupled motion. The nudged elastic band method (NEB) is used to compute the energy barrier to overcome for the GB motion between stable positions in the absence of temperature. The initial and final configurations, before and after the jump respectively, are known. The NEB uses N-replicas of the system obtained by linear interpolation between the initial and final state and tracks the atomic structure evolution through the sampling of a minimum energy path (MEP) (Espinosa and Bao, 2013). The MEP describes the energy variation of the system along a collective reaction coordinate $(\mathrm{RC})$ and provides the saddle point, which corresponds to the climbing replica with the highest energy.

In the present work, 32 replicas of the system are constructed. From the computed MEP for $\Sigma 17(410)$, the saddle point gives an energy barrier of $2.01 \mathrm{eV}$.

Assuming the GB shear-coupled motion is due to the collective glide of the array of dislocations, according to Hull and Bacon (1965) the energy to be provided for the array motion has two contributions: the mechanical work done by the applied load and the thermal activation. As the latter increases, the mechanical contribution decreases since thermal fluctuations assist the array to overcome the barrier, and so the critical stress is reduced as explained in Section 2. The activation energy in the absence of temperature is linear in $\tau_{c}^{0}$, and is given by:

$$
\Delta E_{0}=\tau_{c}^{0} b^{2} l
$$

being $\tau_{c}^{0}$ the required stress to move the array at zero temperature identified as the Peierls-Nabarro stress, $b$ the Burgers vector, and $l$ the length of the line. Eq. (6) estimates the $\Sigma 17(410)$ barrier as $2.20 \mathrm{eV}$, assuming $\tau_{c}^{0}$ equal to $5.45 \mathrm{GPa}$ (see

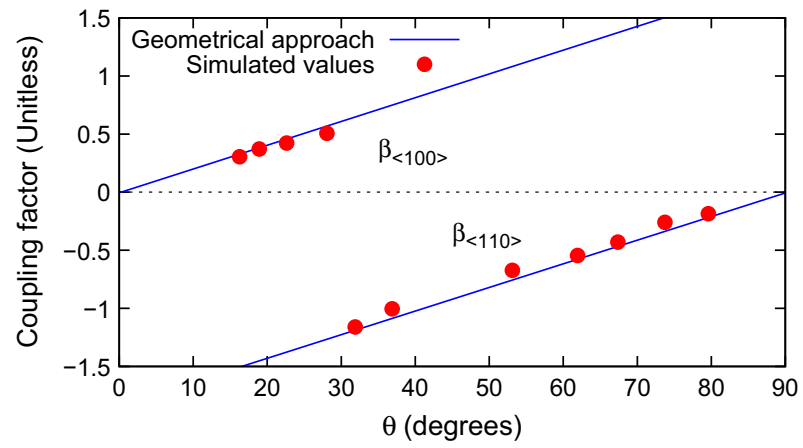

Fig. 8. Coupling factor obtained at $900 \mathrm{~K}$ as function of the tilt angle. Blue lines represent the two branches given by Eqs. (3) and (4). (For interpretation of the references to colour in this figure legend, the reader is referred to the web version of this article.) 

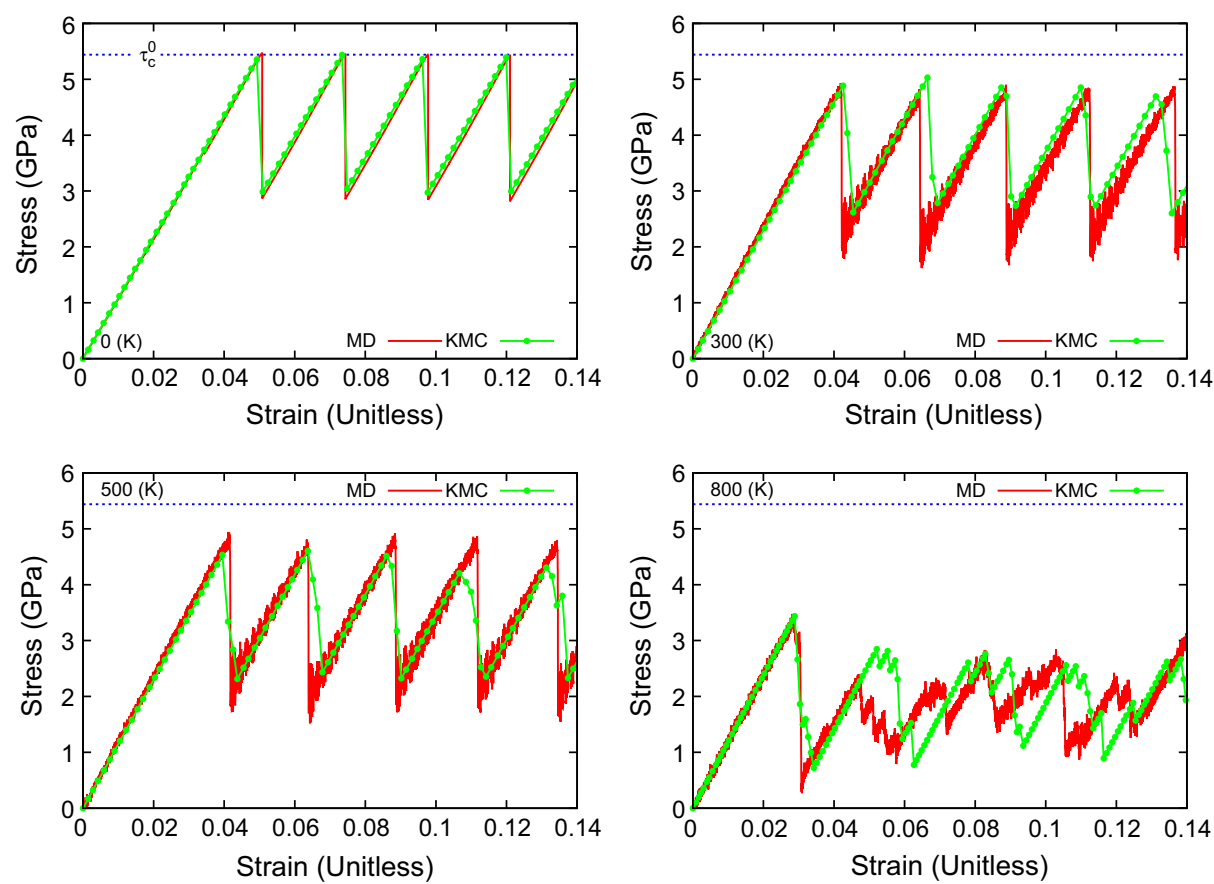

Fig. 9. MD and KMC modelling of GB shear-coupled motion at different temperatures for $\Sigma 17(410)$. Critical stress at zero temperature is identified with a dashed-blue line. (For interpretation of the references to colour in this figure legend, the reader is referred to the web version of this article.)

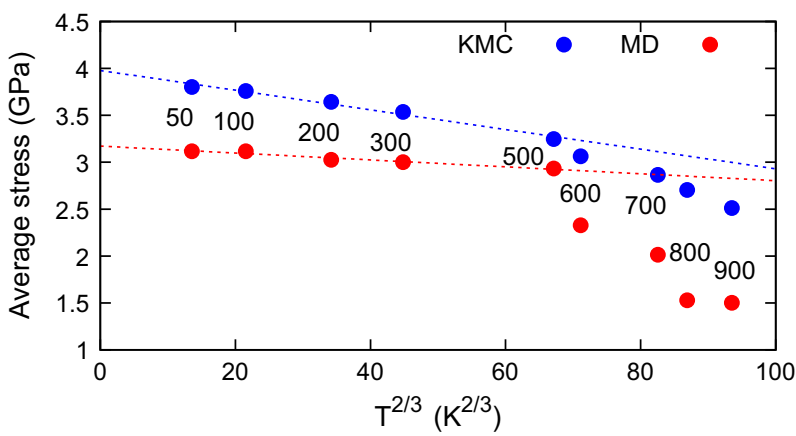

Fig. 10. Averaged stress as function of $T^{2 / 3}$ obtained by MD and KMC simulations. Blue and red lines represent the fit of KMC and MD results, respectively to Eq. (1) for temperatures below $500 \mathrm{~K}$. (For interpretation of the references to colour in this figure legend, the reader is referred to the web version of this article.)

Fig. 3). This value well matches the NEB results of $2.01 \mathrm{eV}$. It should be pointed out the relevance of the latter as input in the KMC modelling of this phenomena in next sections.

\section{Kinetic Monte Carlo}

While using MD, most of the time is spent in describing atomic vibrations, KMC characterizes the system by occasional transitions from state to state. KMC overcomes the time-scale problem by simulating the time evolution of a process occurring at a given known rate.

Coupled motion is a physical process in which the KMC method is applicable. GBs are not considered as a front, instead, the coupled motion is modelled in terms of the single constituent dislocations forming each boundary. By considering individual cores with an associate rate, KMC models the actual MEP: the motion begins with a step of a single unit or a group of them, initiating a disconnection, the first step spreads over the GB and the transition is completed (Rajabzadeh et al., 2013a,b, 2014). 


\subsection{Simulation methodology}

In the present model, the KMC procedure is based on a stochastic algorithm that simulates the evolution of the system from state to state (Voter, 2007). For a given configuration of the GB system, all possible processes are determined, e.g. the jcore jumps, and a list of rates, $r_{j}$, is built. The rates are calculated according to the transition state theory (TST) as:

$$
r_{j}=r_{0} \exp \left(-\frac{\Delta G}{k_{B} T}\right),
$$

being $r_{0}$ the physical attempt frequency calibrated to be $10^{14} \mathrm{~s}^{-1}, \Delta G$ the activation free energy, and having $k_{B} T$ the usual meaning. The total rate of events is calculated as:

$$
R_{i}=\sum_{j=1}^{i} r_{j}
$$

for $i=1$ : $N$, being $N$ the total number of transitions. The actual escape takes place along one of these pathways. By generating a random number $n$ within the range $[0,1)$, the event to perform is selected, e.g. the j-core will jump. As a next step such event is performed, the disconnection is initiated, and the time is increased by:

$$
\Delta t=\frac{1}{R_{N}} .
$$

Once the affected rates are recalculated, the algorithm goes back to the computation of the cumulative function, Eq. (8).

As input of the KMC method, the transition rates are to be known in advance. Such rates are calculated based on the thermodynamics of dislocation motion reported by Hull and Bacon (1965) and previously described in Section 3.4. The energy barrier that each unit sees when it moves is given by:

$$
\Delta G=\Delta E_{0}-\tau V,
$$

being $\Delta E_{0}$ equal to $\Delta E_{N E B}=2.01 \mathrm{eV}, V$ the activation volume for the process calculated in the absence of temperature as $\Delta E_{0} / \tau$ and equal to $59.1 \AA^{3}$, and $\tau V$ the mechanical contribution.

According to Table 2, $\Delta G$ in obtained knowing the stress at the initial step, $t$. As explained above, the event to perform is the discrete transition of a single core, that takes place in $\mathbf{b}$ units. When such transition occurs an amount of energy per unit volume is dissipated, and as a consequence there is a change in the total internal energy of the system which is related with the stress as:

$$
u=\frac{1}{2} \frac{\tau^{2}}{\mu^{e f f}} .
$$

By using Eq. (11), the value of $\tau$ at $t+\Delta t$ is obtained and the algorithm goes back to the computation of $\Delta G$.

\subsection{Simulation results and discussion}

The scaled-up parameters from MD to KMC are $\mu^{\text {eff }}, \tau_{c}^{0}$, b and $\Delta E_{N E B}$, see Table 3. The dimensions and the number of cores of each GB are also introduced as an input. $\Sigma 17(410)$ is used as a model. The stress dependence on strain is computed at different temperatures and compared to MD results as displayed in Fig. 9.

Results obtained via both techniques are in good agreement within a range of temperatures in which the stick-slip behaviour is the dominant response of the GB, seen in Fig. 9. As expected, the critical stress decreases with temperature. Thermal fluctuations reduce the mechanical contribution, allowing the GB to move before $\tau_{c}^{0}$ is reached, identified with a dashed-blue line. For $800 \mathrm{~K}$, the match between MD and KMC is not as clear as at lower temperatures. On the one hand, at such elevated temperature, there is random component in the process that should be taken into account, since there is a finite probability of spontaneous jumps to occur. On the other hand, from MD simulations, $\Sigma 17(410)$ coupled motion was observed to be occasionally interrupted by sliding events, and the noisy behaviour of the stress indicates the transition to other mode, see Fig. 6. The presence of other mechanisms not modelled by KMC is thought to be responsible of such differences. Moreover, it should be taken into account that the $\Sigma 17(410)$ GB switches to the $\langle 100\rangle$ mode at a temperature of $600 \mathrm{~K}$, see Fig. 7. To model the GB motion in terms of the constituent dislocations, the change in the slip plane and the Burgers vector have to be included into the KMC model.

As a test of the agreement in the transition temperature in both models, the average stress obtained by KMC at different temperatures is fit to Eq. (1). Fig. 10 illustrates how the temperature at which the stick-slip behaviour breaks well matches the one obtained via MD. The deviation of the slope between both fittings is evident, however it should be noticed that here the average stress over the whole simulation time is considered, and according to Fig. 9 there is not a perfect match in the stress drop leading to different averaged stress. This mismatch in the stress drop is a limitation of the proposed KMC model, related with the fact that MD addresses the interaction between cores. 
Table 2

KMC algorithm to model the shear-coupled motion of GBs.

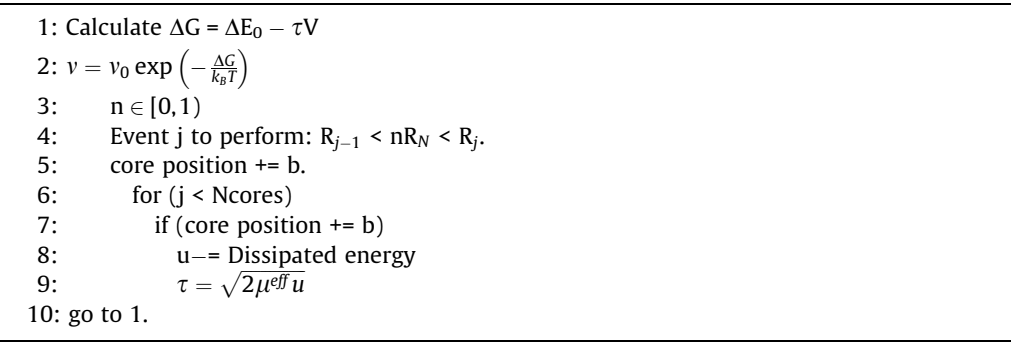

Table 3

Input parameters incorporated to KMC obtained by MD simulations for $\Sigma 17(410)$.

\begin{tabular}{lllll}
\hline$\mu_{M D}^{e f f}(\mathrm{GPa})$ & $\tau_{c}^{0}(\mathrm{GPa})$ & $\mathbf{b}_{\langle 110\rangle}(\AA)$ & $\mathbf{b}_{\langle 100\rangle}(\AA)$ & $\Delta E_{N E B}^{0}(\mathrm{eV})$ \\
\hline 107.76 & 5.45 & 2.49 & 3.52 & 2.01 \\
\hline
\end{tabular}

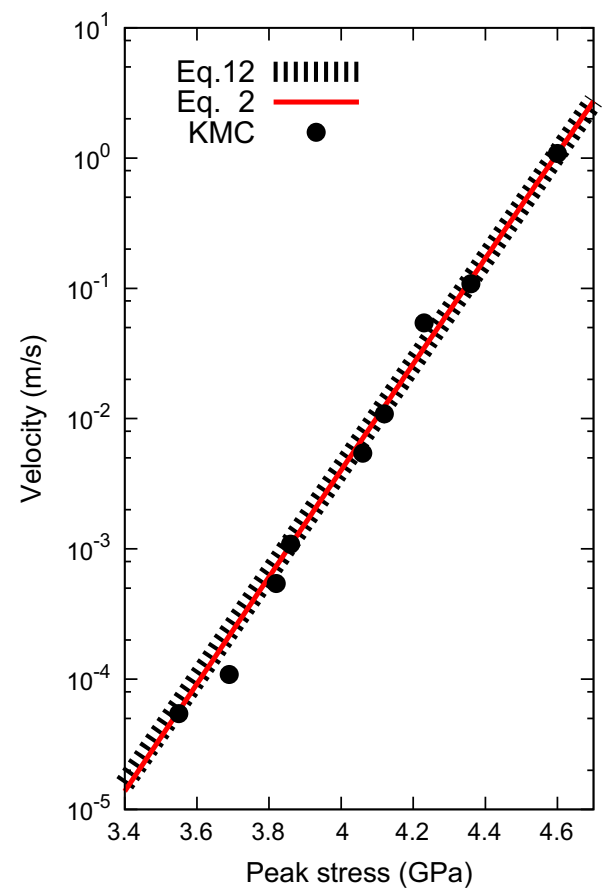

Fig. 11. $\Sigma 17(410)$ velocity in logarithmic scale as function of the averaged peak stress at $400 \mathrm{~K}$. Red line corresponds to the fit to Eq. (2) and black-dashed line to the fit to Eq. (12). (For interpretation of the references to colour in this figure legend, the reader is referred to the web version of this article.)

From Fig. 10, it could be confirmed that KMC restricts the modelling of GB migration to the case of stick-slip regime. This fact may reduce the accuracy of the description of the GB response in the whole range of temperatures. However the implementation of other mechanisms is an existing challenge for future work.

It should be mentioned at this point what is the relevance of KMC in terms of the computational time. MD simulations described in Section 3 take about $14 \mathrm{~h}$, while modelling the same GB response using KMC takes only few seconds. The appeal of the KMC method is that it allows us to simulate larger sample sizes and longer time scales, allowing to overcome the limitations of regular MD, which implies that the imposed velocities are orders of magnitude higher than experiments. By using KMC, the shear velocity range has been greatly expanded, approaching to realistic deformation rates. The KMC model is applied to test the velocity dependence of the critical stress. At a fixed temperature of $400 \mathrm{~K}$, the peak stress is averaged over the whole range of time and plotted versus the imposed velocities within a range from $60 \mu \mathrm{m} / \mathrm{s}$ to $2 \mathrm{~m} / \mathrm{s}$, as shown in Fig. 11 . 
Simulated values are fit to Eq. (2), being $\eta, \tau_{c}^{0}$ and $E_{0}$ adjustable parameters. The last two are compared to values obtained in previous sections in order to test the accuracy of the KMC method at modelling the coupling effect. The corresponding values from the fit in Fig. 11 are $\tau_{c}^{0}=5.63 \mathrm{GPa}$ and $E_{0}=2.01 \mathrm{eV}$, both in good agreement with $\tau_{c}^{0}=5.45 \mathrm{GPa}$ obtained by MD simulations, and $E_{0}=2.01 \mathrm{eV}$ from NEB calculations, respectively.

After exploring the dynamics of coupled motion of GBs by means of MD and extending the model into KMC, a simple relation between the imposed velocity and the peak stress is proposed by direct extension of the thermodynamic approach taken in the KMC model as:

$$
v_{\|}=v \mathbf{b} \exp \left(-\frac{\Delta E_{0}-\tau V}{k_{B} T}\right)
$$

being $v$ the attempt frequency related with vibration events, $\mathbf{b}$ the Burgers vector, $\Delta E_{0}$ the energy barrier in the absence of temperature, $V$ the activation volume for the process, and having $k_{B} T$ the usual meaning. This type of equations, introduced by Kocks et al. (1975) are commonly used in continuum crystal plasticity to link the macroscopic response to thermal activated processes occurring at the atomic scale. The KMC model accounts the thermal activated process assuming discrete dislocation cores. If the total number of cores tends to infinite, the response of the GB system fulfills Eq. (12).

To test this simple relation against atomistic simulations, points obtained at different imposed velocities at a temperature of $400 \mathrm{~K}$ are fit to Eq. (12), as shown in Fig. 11. The adjustable parameters obtained are $V=52.8 \AA^{3}$ and $\Delta E_{0}=1.99 \mathrm{eV}$, both in good agreement with theoretical and NEB values of $59.1 \AA^{3}$ and $2.01 \mathrm{eV}$, respectively.

\section{Concluding remarks}

A bottom-up approach is presented to describe the shear coupled motion in Ni bicrystals. To that end two different atomistic simulation techniques are used: MD and KMC. Although MD is a useful tool in understanding the elementary atomic mechanism that govern the GB migration, simulating realistic sample sizes, boundary conditions and deformation rates are subjected to the limitations of the technique. Using a statistical approach involving KMC methods, the modelling is extended closer to reality by incorporating the output obtained via MD simulations.

At low temperatures, the GB migration exhibits the so-called stick-slip behaviour, characterized by the typical saw-tooth strain dependence of the stress. The stop-and-go motion of the eleven symmetrical tilt GBs has been described in terms of the ratio between the normal motion of the GB and the rigid translation of the adjacent grains.

The motion of the GBs has been also examined at high temperatures, to shed light into the possible changes of the dynamics of GB motion. Identifying the temperature at which the presence of competing mechanisms are observed, is the key to model the pure coupling response in KMC.

Exploring the dynamics of GB shear-coupled motion in terms of the energy leads to identify the mechanism as a thermal activated process (Mishin et al., 2007). The GB moves between equivalent stable positions separated by an energy barrier, which is calculated using the NEB method.

Once the GB structures are fully described focusing on both the energetic and structural characteristics, the output is extended into a KMC model. KMC describes the evolution of the system through occasional transitions from one state to another.

Furthermore, the connection between both techniques has allowed to extend the physical mechanisms of the GB shearcoupled motion into a statistical approach. It should be mentioned how the use of KMC involves an important saving of computational time. The simulation time is reduced from $14 \mathrm{~h}$ spent in MD simulations to $2 \mathrm{~s}$ in KMC. As a consequence, KMC allows to extend the deformation rates by more than 4 orders of magnitude, and imposing grain translation velocities during the shear closer to experimental velocities.

\section{Acknowledgments}

The authors acknowledge partial funding by Abengoa Research. I.M.-B. wants also to acknowledge partial funding from the "Subprograma Ramón y Cajal" fellowship RYC-2012-10639 by the Spanish Ministry of Economy and Competitiveness.

\section{Appendix A. Effective shear modulus for planar GBs: application to [001] STGB contained in bicrystal structures}

Considering the schematic structure of the bicrystal represented in Fig. A.12, the effective elastic tensor is calculated according to Franciosi and Berbenni (2008). In first place, the anisotropic elastic constants of each constituent crystal are obtained by the following transformation rules Gemperlova et al. (1989):

$$
C_{i j k l}=C_{12} \delta_{i j} \delta_{k l}+C_{44}\left(\delta_{i k} \delta_{j l}+\delta_{i l} \delta_{j k}\right)+C_{0} \sum_{s=1}^{3} e_{i}^{(s)} e_{j}^{(s)} e_{k}^{(s)} e_{l}^{(s)},
$$

where $C_{0}=C_{11}-C_{12}-2 C_{44}$, being $C_{0}, C_{11}$ and $C_{14}$ the elastic constant for pure Ni Mishin et al. (1999). $\varphi$ is the rotation angle of [100] around the tilt axis, then $\varphi^{I}=\theta / 2$ and $\varphi^{I I}=-\theta / 2$. The units vectors of the cubic lattice system are: 


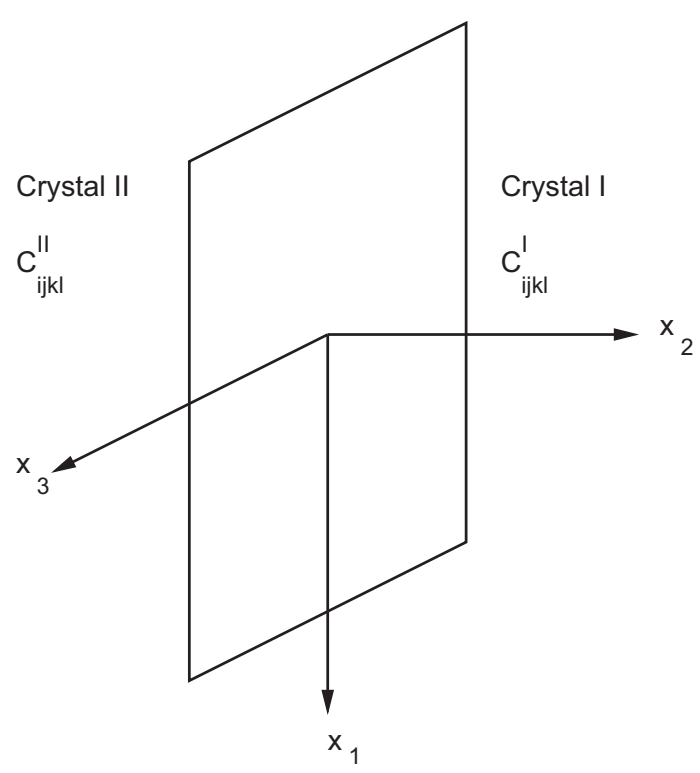

Fig. A.12. Schematic structure of the bicrystal with the coordinate axes used to compute the elastic tensor.

$$
e_{i}^{(1)}=\left(\begin{array}{c}
\cos \varphi \\
\sin \varphi \\
0
\end{array}\right), \quad e_{i}^{(2)}=\left(\begin{array}{c}
-\sin \varphi \\
\cos \varphi \\
0
\end{array}\right), \quad e_{i}^{(3)}=\left(\begin{array}{l}
0 \\
0 \\
1
\end{array}\right) .
$$

Assuming the volume fraction is equal for both constituent crystals, $f^{I}=f^{I I}=0.5$, the transformation field analysis (TFA) is applied for this particular case. The convention proposed by Nye (1957) is adopted, where pairs of subscripts $i j$ and $k l$ are converted to single ones as: $11 \rightarrow 1,22 \rightarrow 2,33 \rightarrow 3,23$ and $32 \rightarrow 4,13$ and $31 \rightarrow 5,12$ and $21 \rightarrow 6$. Referred to the bicrystal structure in Fig. A.12, the in-plane matrix components of the tensor are denoted $P=1,3,5$ and the out-plane are $A=2,4,6$. As a result, the effective elastic modulus $C_{i j k l}^{\text {eff }}$ :

$$
\left.\left.\left(\begin{array}{c}
\Sigma_{A} \\
\Sigma_{P}
\end{array}\right)=\begin{array}{ll}
C_{A A}^{e f f} & C_{A P}^{e f f} \\
C_{P A}^{e f f} & C_{P P}^{e f f}
\end{array}\right)\left(\begin{array}{c}
E_{A} \\
E_{P}
\end{array}\right) \rightarrow C_{i j k l}^{e f f}=\begin{array}{ll}
C_{A A}^{e f f} & C_{A P}^{e f f} \\
C_{P A}^{e f f} & C_{P P}^{e f f}
\end{array}\right) .
$$

By using the matrix forms of $C_{A A}^{\text {eff }}, C_{A P}^{\text {eff }}, C_{P A}^{\text {eff }}$ and $C_{P P}^{\text {eff }}$ reported by Franciosi and Berbenni (2008), and according to Nye convention, the effective shear modulus is given by:

$$
\left.\left.\mu^{e f f}=\frac{1}{2} C_{66}^{I}-\frac{\left(C_{62}^{I}\right)^{2}}{C_{22}^{I}}\right)+\frac{1}{2} C_{66}^{I I}-\frac{\left(C_{62}^{I I}\right)^{2}}{C_{22}^{I I}}\right)
$$

\section{References}

Berbenni, S., Paliwal, B., Cherkaoui, M., 2013a. Generalized Continua as Models for Materials, vol. 22. Springer, Berlin Heidelberg.

Berbenni, S., Paliwal, B., Cherkaoui, M., 2013b. A micromechanics-based model for the shear-coupled grain boundary migration in bicrystals. Int. J. Plast. 44, 68-94.

Bieler, T., Eisenlohr, P., Roters, F., Kumar, D., Mason, D., Crimp, M., Raabe, D., 2009. The role of heterogeneous deformation on damage nucleation at grain boundaries in single phase metals. Int. J. Plast. 25, 1665.

Bilby, B.A., 1955. Types of dislocation sources. Phys. Soc. London, 124-133.

Cahn, J.W., Taylor, J., 2004. A unified approach to motion of grain boundaries, relative tangential translation along grain boundaries, and grain rotation. Acta Mater. 52 (16), 4887-4898.

Cahn, J.W., Mishin, Y., Suzuki, A., 2006. Coupling grain boundary motion to shear deformation. Acta Mater. 54, 4953-4975.

Dahlberg, C., Faleskog, J., Niordson, C., Legarth, B., 2013. A deformation mechanisms map for polycrystals modeled using strain gradient plasticity and interfaces that slide separate. Int. J. Plast. 43, 177.

Du, N., Qi, Y., Krajewski, P., Bower, A., 2010. Aluminum grain boundary sliding enhanced by vacancy diffusion. Acta Mater. 58, 4245-4252.

Dvorak, G.J., 1990. On uniform fields in heterogeneous media. Proc. Roy. Soc. London A 431, 89-110.

Espinosa, H., Bao, G., 2013. Nano and Cell Mechanics: Fundamentals and Frontiers. John Wiley and Sons.

Faken, D., Jonsson, H., 1994. Systematic analysis of local atomic structure combined with 3D computer graphics. Comput. Mater. Sci. 2 (279).

Farkas, D., 2000. Atomistic theory and computer simulation of grain boundary structure and diffusion. J. Phys. Conds. Matter. 12 , 497.

Franciosi, P., Berbenni, S., 2008. Multi-laminate plastic strain organization for non-uniform TFA modeling of polycrystal regularized plastic flow. Int. J. Plast. 24, 1549-1580.

Frank, F.C., 1953. The resultant content of dislocation in an arbitrary intercrystalline boundary. In: Symposium on the Plastic Deformation of Crystalline Solids. Carnegie Institute of Technology, Pittsburgh, pp. 150-154. 
Frolov, T., 2014. Effect of interfacial structural phase transitions on the coupled motion of grain boundaries: a molecular dynamics study. Appl. Phys. Lett. 104 (211905).

Garg, A., 1995. Escape-field distribution for escape from a metastable potential well subjected to a steadily increasing bias field. Phys. Rev. B 51 (15592). Gemperlova, J., Paidar, V., Kroupa, V., 1989. compatibility stresses in deformed bicrystals. Czech. J. Phys. B 39, 427.

Goryaka, T., Modolov, D.A., Gottstein, G., 2009. Stress-driven of symmetrical $\langle 100\rangle$ tilt grain boundaries in Al bicrystals. Acta Mater. 57, $5396-5405$.

Goryaka, T., Modolov, K., Modolov, D.A., Gottstein, G., 2011. Concurrent grain boundary motion and grain boundary rotation under an applied stress. Acta Mater. 59, 5674.

Hirth, J., 1972. The influence of grain boundaries on mechanical properties. Metall. Mater. Trans. B 3 (12), 3047.

Homer, E., Foiles, S., Holm, E., Olmsted, D., 2013. Phenomenology of shear-coupled grain boundary motion in symmetric tilt and general grain boundaries. Acta Mater. 61, 1048.

Huang, B., Shang, J., Liu, Z., Chen, Y., 2014. Atomic simulation of bcc niobium $\Sigma 5\langle 100\rangle\{310\}$ grain boundary under shear deformation. Acta Mater. 77, 258.

Hull, D., Bacon, D., 1965. Introduction to Dislocations. Pergamon Press.

Ivanov, V.A., Mishin, Y., 2008. Dynamics of grain boundary motion coupled to shear deformation: an analytical model and its verification by molecular dynamics. Phys. Rev. B 78 (064106).

Kocks, U., Argon, A., Ashby, F., 1975. Thermodynamics and Kinetics of Slip, vol. 19. Pergamon Press.

Kronenberg, M., Wilson, F., 1947. Secondary recrystallization in copper. Trans. Met. Soc. AIME 185, 501.

Li, C., Edwards, E., Washburn, J., Parker, E., 1953. Stress-induced movements of crystal boundaries. Acta Metall. 1, 223.

Mishin, Y., Farkas, D., Mehl, M.J., Papaconstantopoulos, D., 1999. Interatomic potentials for monoatomic metals from experimental data and ab initio calculations. Phys. Rev. B 59 (3393).

Mishin, Y., Suzuki, A., Uberuaga, B.P., Voter, A.F., 2007. Stick-slip behaviour of gbs studied by accelerated md. Phys. Rev. B 75 (224101).

Mishin, Y., Asta, M., Li, J., 2010. Atomistic modeling of interfaces and their impact on microstructure and properties. Acta Mater. 58, 115-117.

Nye, J., 1957. Physical Properties of Crystals. Clarendon Press, Oxford.

Ohashi, T., Barabash, R., Pang, J., Ice, G., Barabash, O., 2009. X-ray microdiffraction and strain gradient crystal plasticity studies on geometrically necessary dislocations near a Ni bicrystal grain boundary. Int. J. Plast. 25, 920.

Plimpton, S.J., 1995. Fast parallel algorithms for short-range molecular dynamics. J. Comput. Phys. 117, 1-19.

Rajabzadeh, A., Legros, M., Combe, N., Mompiou, F., Modolov, D.A., 2013a. Evidence of gb dislocation step motion associated to shear coupled gb migration. Philos. Mag. 93 (10-12), 1299-1316.

Rajabzadeh, A., Mompiou, F., Le, M., Combe, N., 2013b. Elementary mechanisms of shear-coupled grain boundary migration. Phys. Rev. Lett. 110 (265507).

Rajabzadeh, A., Mompiou, F., Lartigue-Korinek, S., Combe, N., Legros, M., Modolov, D.A., 2014. The role of disconnections in deformation-coupled grain boundary migration. Acta Mater. 77, 223.

Read, W., Shockley, W., 1950. Dislocation models of crystal boundaries. Phys. Rev. B 78, 275.

Sang, Y., Dube, M., Grant, M., 2001. Thermal effects on atomic friction. Phys. Rev. Lett. 87 (174301).

Sansoz, F., Molinari, J.F., 2005. Mechanical behaviour of Sigma tilt grain boundaries in nanoscale Cu and Al: a quasicontinuum study. Acta Mater. 52, 19311944.

Schäfer, J., Albe, K., 2012. Competing deformation mechanisms in nanocrystalline metals and alloys: coupled motion versus grain boundary sliding. Acta Mater. 60, 6076.

Spearot, D., Jacob, K., McDowell, D., 2007. Dislocation nucleation from bicrystal interfaces with dissociated structures. Int. J. Plast. $23,143$.

Taupin, V., Capolungo, L., Fressengeas, C., 2014. Disclination mediated plasticity in shear-coupled boundary migration. Int. J. Plast. 53, 179.

Tschopp, M., McDowell, D., 2008. Dislocation nucleation in $\Sigma 3$ asymmetric tilt grain boundaries. Int. J. Plast. $24,191$.

Tucker, G., Zimmerman, J., McDowell, D., 2010. Shear deformation kinematics on bicrystalline grain boundaries in atomistic simulations. Modelling Simul. Mater. Sci. Eng. 18 (0150002).

Turley, J., Sines, G., 1971. The anisotropy of Young modulus, Shear modulus and Poisson ratio in cubic materials. J. Phys. D: Appl. Phys. 4.

Voter, A., 2007. Introduction to Kinetic Monte Carlo Method, vol. 253. NATO Science Series.

Warner, D., Roters, F., Molinari, J., 2006. Atomistic based continuum investigations of plastic deformation in nanocrystalline copper. Int. J. Plast. $22,754$. Wulfinghoff, S., Bayerschen, E., Böhlke, T., 2013. A gradient plasticity grain boundary yield theory. Int. J. Plast. 51, 33. 